\title{
CLINICAL AND HISTOPATHOLOGICAL CORRELATION IN TUBERCULOSIS OF SPINE BIOPSIES
}

Muhammad Shahbaz Amin ${ }^{a}$, Seema Butt ${ }^{a}$, Abdullah Shahbaz ${ }^{b}$, Majid Zaheer ${ }^{c}$, Ashfaq Ahmed ${ }^{c}$, Shazia Nilofer Ibnerasa $^{d}$

${ }^{a}$ Associate Professor, Department of Pathology, Lahore Medical \& Dental College Lahore.

${ }^{\mathrm{b}}$ Medical Student at Rahbar Medical \& Dental College Lahore.

'Post-Graduate Resident Department of Orthopedic \& Spine Surgery, Ghurki Trust Teaching Hospital Lahore.

${ }^{d}$ Professor Department of Pathology Lahore Medical \& Dental College Lahore.

\section{ABSTRACT:}

BACKGROUND \& OBJECTIVE: The tuberculosis (TB) of the spine is causing permanent deformities since long time. Earliest most possible accurate diagnosis is required to treat either TB or to exclude other lesions to avoid permanent disability.

METHODOLOGY: This is a descriptive study conducted at pathology department of Lahore Medical \& Dental College (LM\&DC), Lahore from January 2017 to December 2019. All the spine biopsies received in Pathology department were included in the study irrespective of age and sex. Each biopsy was grossly and microscopically examined and reported by a histopathologist.

RESULTS: Out of 386 specimens received, 189 (48.9\%) were clinically diagnosed as tuberculosis. Out of these 189 cases, 127 were confirmed on histopathological examination showing clinicopathological correlations in $67.19 \%$ of cases. The rest $62(32.80 \%)$ of the cases were reported as chronic non specific inflammation (38), degenerative changes (11), metastatic tumor (04), and atypical cell infiltrate (02), plasma cell neoplasm (01), Non Hodgkin Lymphoma (01) and other lesions (05) which all were benign. Out of the total 386 spine biopsies, 154 (39.89\%) were histopathologically proven for tuberculosis. From these 154 histologically proven cases, 127 were clinically suspected cases of Tuberculosis. No clinical diagnosis was provided in 02 patients. The rest of 25 patients were clinically labeled as pathological lesion without specific diagnosis.

CONCLUSION: Histopathology should be considered as the most reliable tool for spinal tuberculosis to minimize permanent disability associated with wrong management based on potentially erroneous clinical diagnosis.

KEYWORDS: Spine, Spinal tuberculosis, Histopathology, Diagnosis.

\section{How to cite this:}

doi: https://doi.org/10.37723/jumdc.v12i1.513

Amin MS, Butt S, Shahbaz A, Zaheer M, Ahmed A, Ibnerasa SN. CLINICAL AND HISTOPATHOLOGICAL CORRELATION IN TUBERCULOSIS OF SPINE BIOPSIES. jumdc. $2021 ; 12(1): 30-35$.

doi: https://doi.org/10.37723/jumdc.v12i1.513

This is an Open Access article distributed under the terms of the Creative Commons Attribution License (http://creativecommons.org/licenses/by/4.0), which permits unrestricted use, distribution, and reproduction in any medium, provided the original work is properly cited. 


\section{INTRODUCTION:}

Tuberculosis still remains a disease that causes distress and suffering to the patient even in this era of modern medical science and demands that this global issue should be properly addressed ${ }^{[1]}$. It is a chronic disease which usually presents with low grade fever and weight loss. It is one of the major cause of death in the world. The disease is responsible for about 2 million deaths from approximately 8 million recognized cases worldwide ${ }^{[2]}$. World Health Organization (WHO) Global Tuberculosis Report of 2016 listed South Africa as having the highest incidence of tuberculosis (TB) per capita in the world, with an estimated incidence of 8$16: 100,000$, almost $95 \%$ of cases diagnosed as tuberculosis, are in developing countries ${ }^{[3]}$.

Once thought to be an endemic disease in underprivileged region/area of developing countries, tuberculosis has now become a problem of international concern spreading all over the world due to easy and frequent cross country travelling. In 1993, WHO declared the TB as a major health problem in developing countries. The history of spinal TB dates back to 1779; and Tuberculous Spondylitis was first reported by Sir Percival Pott ${ }^{[4]}$. Spinal tuberculosis or spondylitis, is the most common and the most serious form of skeletal tuberculosis ${ }^{[5]}$. It refers to an infection of the intervertebral disk, the vertebral body itself or the posterior vertebral arch, by Mycobacterium tuberculosis, with or without involvement of the spinal canal ${ }^{[6]}$. Thoracic region of the spine is usually the most common part which gets involved with the disease ${ }^{[5]}$. When the body of the spine is involved, instability of the affected spine can occur with anterior or lateral displacement of the vertebra. This results in physical deformity and also carries a risk of cord compression, especially in children and elderly resulting in paraplegia, and pulmonary insufficiency ${ }^{[2,5]}$.

Mycobacterium tuberculosis, the causative organism of TB is a slow-growing, acid-fast aerobic bacillus. This bacillus tends to remain dormant for prolonged periods and multiplies every 15 to 20 hours in aerobic conditions whenever favorable. The primary site of infection can be in the lungs, lymph nodes of the mediastinum, mesentery, gastrointestinal
Corresponding Author:

Dr. Shahbaz Amin

Associate Professor, Department of Pathology, Lahore Medical \& Dental College Lahore.

Email: shahbazamin415@gmail.com

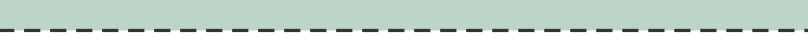

tract, genitourinary system, or any other viscera. Spinal infection is almost always secondary and is caused by the hematogenous spread of this bacillus from a primary site ${ }^{[4]}$.

Spinal TB is usually insidious in onset. The disease might take from 2 weeks to several years from the onset of symptoms before the diagnosis can be achieved ${ }^{[5]}$.

A patient with spinal tuberculosis seldom presents with symptoms (such as cough, sputum, fever, weight loss, or night sweating), which are common complaints in pulmonary tuberculosis, and this results in a delayed diagnosis. Back pain is usually the most common presenting symptom, followed by night sweats, weight loss, and low-grade fever [7].

Lack of early symptoms and insidious onset make early diagnosis tricky. Physicians and surgeons usually rely on a several modalities for the diagnosis of spinal tuberculosis. These include an examination of clinical signs and symptoms, radiological investigations, microbiological tests, metabolic product detection (interferon-Y [IFN-Y] test), polymerase chain reaction (PCR), and tissue biopsy. Most of these procedures are invasive and complex and diagnosis can take several days. There is currently no single diagnostic method that can detect all tuberculosis types and cases ${ }^{[2]}$.

The Growth of TB bacillus on specific culture media, on inoculation of infected tissue, has been considered as the gold standard for the diagnosis of spinal tuberculosis. However, due to its very poor sensitivity, histopathological studies demonstrating epithelioid granulomas and staining of smears to identify acid-fast bacilli (AFB) are considered as a reference standard for all other diagnostic modalities ${ }^{[4]}$.

Depending only on clinical and radiological features many lesions can be missed resulting in delayed diagnosis thus causing irreversible damage and serious complications. Although plain X-ray, MRI, and CT scans are used for the 
detection of spinal pathologies; none of these imaging techniques are reliable with certainty to distinguish between infectious and neoplastic processes. So histopathology is mandatory to confirm the diagnosis of tuberculosis and to rule out other pathologies including malignancies ${ }^{[7]}$. In developing countries for early detection and differentiation of spine tuberculosis from other similar lesions, clinical features and plain radiographs are the main diagnostic tools for this disease ${ }^{[8,9,10]}$.

Although there is a global consensus about the importance of early identification of spinal tuberculosis but literature is still deficient in guiding the primary health care practitioners to adopt a good clinical approach and use of an appropriate diagnostic tool in resource poor setting for prompt diagnosis and management ${ }^{[3]}$. The literature is also deficient on the frequency of lesions that mimic spinal tuberculosis ${ }^{[2,8]}$.

Taking histopathological biopsy as a reference standard, the study was planned to determine the accuracy of clinical diagnosis of cases of spinal tuberculosis by correlating it with histopathological biopsy report. This study will highlight the importance of histopathological biopsy as an adjunct to clinical signs and symptoms suggestive of TB. It will also differentiate tuberculosis from other malignant and benign lesions that resemble this common and disabling disease on clinical and/or radiological findings. It will also provide the basic knowledge for the future to determine the relative reliability of different diagnostic modalities and to establish a more accurate diagnostic tool in spinal tuberculosis.

\section{METHODOLOGY:}

This descriptive cross-sectional study was conducted in the Pathology department of Lahore Medical and Dental College (LM\&DC) Lahore for 03 years i.e., from January 2017 to December 2019. After ethical approval from IRB-LM\&DC, data was collected.

This study was carried out on 386 biopsies of the spine that were received in the Pathology Department of LM\&DC Lahore. All the cases of spine biopsy irrespective of age, sex, and origin were included in this study. Patient particulars, history, clinical information, investigation, and all other information provided on the request form were entered on a separate clinical performa for each biopsy. Most of the biopsy material was received from the spine centre of Ghurki Trust Teaching Hospital (GTTH), one of the largest spine centre, where patients come from all over Pakistan. The specimens received were all fixed in $10 \%$ formalin. The Gross examination was done and representative sections of the lesion were taken, which were processed in an automated tissue processor. Initially, routine staining with Hematoxylin and Eosin was done. $\mathrm{Z} \mathrm{N}$ staining was done in those cases where tuberculosis was suspected on microscopy. The slides were initially examined by residents and then the concerned consultant examined every case personally for final diagnosis and reporting. Intra-departmental consultation was done where required.

Data was entered in SPSS-22 package. Descriptive statistics was used to analyze the data, which was presented in the form of tables.

\section{RESULTS:}

Total 386 cases of spine biopsy received were included in this study. The age of patients ranged from 03 to 72 years with the mean age being 35 years. The majority of patients were of 21-50 years of age. No sex predilection was observed, both the female and the male patients were equally affected by tuberculosis of spine. Almost all of the cases were from thoraco-lumbar spine; thoracic region was involved more than lumbar area. Clinically tuberculosis was suspected in 189 (48.96\%) cases from the total 386 spine biopsies. From these 189 clinically suspected tuberculous biopsies $127(67.19 \%)$ were confirmed on histopathological examination as well showing clinicopathological correlations in $67.19 \%$ of cases. The rest of $62(32.80 \%)$ cases were reported as chronic nonspecific inflammation 38 $(20.10 \%)$, degenerative changes11 (5.82\%), metastatic tumor $04(2.11 \%)$, atypical cell infiltrate 02(1.05\%), plasma cell neoplasm $01(0.52 \%)$, Non-Hodgkin's Lymphoma $01(0.52 \%)$ and other lesions $05(2.64 \%)$ all of those were benign (Table-I). 
Table-I: Histopathological diagnoses in spine biopsies clinically suspected as tuberculosis.

\begin{tabular}{|c|c|c|l|}
\hline No & Histopathological Diagnosis $(\mathbf{n = 1 8 9 )}$ & No of Cases & \multicolumn{1}{|c|}{$\%$} \\
\hline $\mathbf{1}$ & Tuberculosis & 127 & 67.19 \\
\hline $\mathbf{2}$ & Chronic Non Specific inflammation & 38 & 20.10 \\
\hline $\mathbf{3}$ & Degenerative Changes & 11 & 05.82 \\
\hline $\mathbf{4}$ & Metastatic tumor & 04 & 02.11 \\
\hline $\mathbf{5}$ & Atypical cell Infiltrate & 02 & 01.05 \\
\hline $\mathbf{6}$ & Plasma cell neoplasm & 01 & 00.52 \\
\hline $\mathbf{7}$ & Non Hodgkin Lymphoma & 01 & 00.52 \\
\hline $\mathbf{8}$ & Others & 05 & 02.64 \\
\hline
\end{tabular}

Of the total 386 cases of spine biopsy, 154 (39.89\%) cases were histopathologically diagnosed as tuberculous. From these 154 histopathologically reported cases of tuberculosis of spine biopsies 127 were also clinically suspected cases of tuberculosis. No provisional diagnosis was provided in 02 patients. The rest of the 25 patients were clinically labeled as pathological lesions without any specific provisional diagnosis (Table-II).

Table-II: Clinical diagnoses in histopathologically proven tuberculous spine biopsies:

\begin{tabular}{|c|c|c|c|}
\hline No & Clinical Diagnoses $(\mathbf{n = 1 5 4 )}$ & No of Cases & \% \\
\hline $\mathbf{1}$ & Tuberculosis & 127 & 82.46 \\
\hline $\mathbf{2}$ & Pathological lesion & 25 & 16.23 \\
\hline $\mathbf{3}$ & No diagnosis & 02 & 01.29 \\
\hline
\end{tabular}

\section{DISCUSSION:}

Skeletal tuberculosis accounts for $10 \%$ of extrapulmonary tuberculosis and about $50 \%$ of Skeletal tuberculosis involves the spine. Predisposing factors include malnutrition, diabetes, drug abuse, alcohol, and other chronic diseases. Symptoms are insidious and can be there for months to years before it is suspected by clinical findings and history of contact with tuberculous patients. The radiological/imaging methods along with laboratory investigations like ESR and Mantoux test are usually reliable for diagnosis ${ }^{[7]}$. In a study, it was observed that most of their patients were young with a mean age of 34 years ${ }^{[11]}$. In other studies, most patients were young adults in the $3 r d$ to 5 th decades of life ${ }^{[12,}$ ${ }^{13]}$. In our study, the mean age was 35 years which coincides with previous studies. Our study revealed that thoraco-lumbar spine was the most commonly affected region of spine by tuberculosis, with a predilection for thoracic than lumbar area. In other studies the thoracolumbar involvement was maximum but there were more cases in which the lumbar vertebrae were affected by $\mathrm{TB}^{[14,15]}$. Early diagnosis and management of spinal tuberculosis saves the patient from severe disabling complications requiring prolonged and interventional management and this can only be achieved by a multidisciplinary approach involving radiology and histopathology along with clinical assessment. Laboratory investigations like culture sensitivity and Polymerase chain reaction (PCR) are very helpful in diagnosis but the late and insensitive result of culture sensitivity and low specificity of PCR are major limitations ${ }^{[13,16]}$. The malignant entities of spine like multiple myeloma and metastatic tumor are also very similar to tuberculosis on clinicoradiological assessment which cannot be 
relied upon for definite diagnosis in such cases ${ }^{[13]}$. Tuberculosis and other nontuberculosis lesions are very frequently encountered in the spine and can be missed, misdiagnosed, or mistaken if a definite diagnosis has relied on clinicoradiological findings only which will result in delayed management of malignant lesions with grave effects ${ }^{[1,13,17,18]}$. The spinal TB is very likely to be misdiagnosed due to lack of specific clinical features. The false-negative results of the laboratory tests and imaging studies further increase the possibility of missing tuberculosis. The early diagnosis and management of Spinal TB by drugs reduce the surgical intervention and economic burden. Active tuberculosis is treated with drugs. The surgical intervention in spinal tuberculosis is done for getting biopsy material, debridement, and halting the deformity. In old healed lesions, surgery is required for reinforcement of stability and correction of disabling deformity of the spine ${ }^{[4,11]}$. The gold standard for diagnosis should be isolation and culture of Mycobacterium tuberculosis but slow and insensitive results are its limitations ${ }^{[13]}$. PCR is simple, sensitive, and quick but has poor specificity ${ }^{[13]}$. A quick and most reliable diagnostic tool for early and most possibly accurate diagnosis was needed to reduce the disability by spinal TB.

Watts and Life so and othershave observed that the management of spinal tuberculosis should be based on histopathological diagnosis ${ }^{[4,11,13]}$. The result of our study concurs with previous studies in suggesting that histopathological examination of biopsy should be considered as the most reliable diagnostic tool for spinal tuberculosis.

\section{CONCLUSION:}

The study on 386 spine biopsies revealed that tuberculosis is the most common pathological lesion in $154(39.89 \%)$ cases with a predilection for the thoracic region. From 189 clinically tuberculous cases, clinicalhistopathological correlation was found in 127 $(67.19 \%)$ cases. The rest of $62(32.81 \%)$ cases with poor clinical-histopathological correlation were clinically misdiagnosed as tuberculosis which on histopathology turned out to be malignant lesions like plasma cell tumor, lymphoma, and secondaries besides benign entities. So reliance only on clinical diagnosis may lead to grave disability because of inappropriate management based on clinical diagnosis which may be erroneous. Histopathological diagnosis should be considered as the most reliable diagnostic tool in spinal tuberculosis.

\section{ACKNOWLEDGEMENT:}

I acknowledge the hard work of Dr. Sadia Iqbal, Demonstrator and, Dr. Muhammad Haseeb Ayub, Resident Pathology Department LM\&DC Lahore who followed these cases meticulously to compile the data. I cannot ignore the assistance rendered by Mr. Asif Hussain and Mr. Amjad Ali (laboratory technicians) in the gross examination and tissue processing for good slide preparation and other technical supports.

CONFLICT OF INTEREST: All authors disclose no conflict of interest.

\section{GRANT SUPPORT AND FINANCIAL DISCLOSURE: None}

\section{REFERENCES:}

1. Moon MS, Kim SS, Moon HL, Kim DH. Mycobacterium Tuberculosis in Spinal Tuberculosis. Asian Spine Journal. 2017; 11(1):138-149.

2. Moon MS. Tuberculosis of spine: current views in diagnosis and management. Asian Spine Journal. 2014; 8(1):97-111. Doi:10. 4184/asj.2014.8.1.97

3. Galloway KM, Parker R. Could an increase in vigilance for spinal tuberculosis at primary health care level, enable earlier diagnosis at district level in a tuberculosis endemic country?. African journal of primary health care \& family medicine. 2018; 10(1):1-9. Doi: 10.4102/phcfm. v10i1.1666

4. Rajasekaran S, Soundararajan DCR, Shetty AP, Kanna RM. Spinal Tuberculosis: Current Concepts. Global Spine Journal. 2018; 8(4 Suppl):96S-108S Doi: $10.1177 / 2192568218769053$

5. Patankar AP. Tuberculosis of spine: An experience of 30 cases over two years. Asian Journal of Neurosurg. 2016;

JUMDC Vol. 12, Issue 1, January-March 2021 
$11(3): 226-231 . \quad$ Doi: $10.4103 / 1793-$ 5482.145085

6. Procopie I, Popescu EL, Ple?ea RM, Doroban?u M, Mure?an RF, Ursulescu CVL et al., Clinical-Morphological Aspects in Spinal Tuberculosis. Current Health Sciences Journal. 2018; 44(3):250-260.

7. Wang P, Liao W, Cao G, Jiang Y, Rao J, Yang Y. Characteristics and Management of Spinal Tuberculosis in Tuberculosis Endemic Area of Guizhou Province: A Retrospective Study of 597 Patients in a Teaching Hospital. BioMed Research International. 2020: 1468457 Doi:10.1155/2020/1468457

8. Özturk AM, Yener C, Tasbakan Isikgoz M: current concepts on spinal tuberculosis. Journal of Turkish Spinal Surgery. 2020; 31(1):60-63, Doi: 10.4274/jtss.galenos. 2020.31

9. Nguyen TT, Thelen JC, Bhatt AA. Bone up on spinal osseous lesions: A Case Review series. Insights into Imaging. 2020; 11(80). Doi: 10.1186/s13244-02000883-6

10. Sharif HS, Morgan JL, Alshahed MS, Al Thagafi MY. Role of CT and MRI in management of tuberculous spondylitis. Radiologic Clinics of North America.1995; 33(4):787-804.

11. Pu F, Feng J, Yang L, Zhang L, \& Xia P. Misdiagnosed and mismanaged atypical spinal tuberculosis: A case series report. Experimental and Therapeutic Medicine. 2019; 18(5): 3723-3728. Doi: 10.3892/etm.2019.8014

12. Jun W, Bin S, XiaopengL, Jianmin S, Lin S, WenTao et al.Migration inhibitory factor in spinal tuberculosis: $-173 \mathrm{G} / \mathrm{C}$ polymorphisms, and transcript and protein levels in a northern province of China. Medicine: 2020; 99(30): 21331 Doi: 10.1097/MD.0000000000021331

13. Shaikh SI, Chauhan AA, Patel U. An analysis of clinico-radiological and histopathological correlation in Tuberculosis of spine. National Journal of Medical Research. 2013; 3(3):198-202.

14. Wang B, Kong L, Zhu Z ,Gao W, Guo H, Wang $X$ et al., Recurrent complex spinal tuberculosis accompanied by sinus tract

formation: causes of recurrence and clinical treatments. Scientific Report. 2018; 8(1):6933. Doi:10.1038/s41598018-25142-z

15. Madjid TH, Ardhi I, Permadi W, Susiarno $\mathrm{H}$, Krisnadi SR, Wirakusumah FF, et al. Correlation of Clinical Features, Laboratory Finding, and Pelvic Ultrasonography of Pulmonary Tuberculosis Women with Infertility. International Journal of General Medicine. $2019 ; \quad 12: 485-489$ Doi:10.2147/IJGM.S228464

16. Yin $\mathrm{XH}$, Liu ZK, Hao D. The reasons and clinical treatments of postoperative relapse of Pott's disease. Medicine. 2018; 97(39):e12471. Doi:10.1097/MD. 0000000000012471

17. Chen $\mathrm{CH}$, Chen YM, Lee CW, Chang YJ, Cheng $\mathrm{CY}$, Hung JK. Early diagnosis of spinal tuberculosis. Journal of the Formosan Medical Association.2016; 115(10): 825-836. Doi:10.1016/j.jfma. 2016.07.00

18. Dunn RN, Ben Husien M. Spinal tuberculosis: review of current management. The Bone \& Joint Journal. $2018 ; 100-B$ ( 4): $425-431$. Doi: $10.1302 / 0301-620 X .100 B 4 . B J]-$ 2017-1040.R1

\section{Author's Contribution:}

Muhammad Shahbaz Amin: Conception \& Design the study.

Seema Butt: Data collection and analysis.

Abdullah Shahbaz: Drafting and Editing. Majid Zaheer: Proof reading \& Reference collection.

Ashfaq Ahmed: Proof reading \& Reference collection.

Shazia Nilofer Ibnerasa: Critical review and final approval.

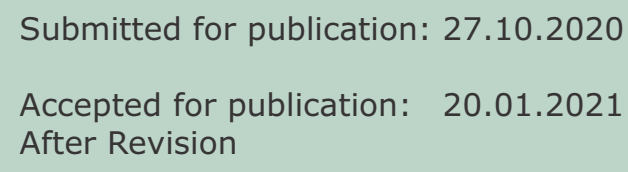

